\title{
AN ELEMENTARY EXAMPLE OF A BANACH SPACE NOT ISOMORPHIC TO ITS COMPLEX CONJUGATE
}

\author{
N.J. KaLton \\ UNIVERSity OF MisSOURI-COLUmbia
}

\begin{abstract}
.
We give a simple and explicit example of a complex Banach space which is not isomorphic to its complex conjugate, and hence of two real-isomorphic spaces which are not complex-isomorphic.
\end{abstract}

If $X$ is a complex Banach space, its complex conjugate $\bar{X}$ is defined to be the space $X$ equipped with the alternative scalar multiplication $\alpha \otimes x=\bar{\alpha} x$. In [1] Bourgain gave the first example of a complex Banach space which is not isomorphic to its complex conjugate, thereby also producing an example of two Banach spaces which are isomorphic as real Banach spaces, but not isomorphic as complex Banach spaces; see also the work of Szarek [5] and Mankiewicz [4]. The approach of all these authors was probabilistic, and no explicit example has been constructed. The aim of this note is to give an elementary explicit construction of a Banach space $X$ so that $X$ and $\bar{X}$ are non-isomorphic. Our example has the further advantage of being, in a certain sense, entirely natural.

The space $X$ we construct is a twisted Hilbert space, i.e. $X$ has a closed subspace $E$ so that $E$ and $X / E$ are Hilbertian; this further implies $X$ is hereditarily Hilbertian.

Let $\omega$ denote the space of all complex-valued sequences, $x=(x(n))_{n=1}^{\infty}$. We let $e_{n}$ be the canonical basis vectors. Suppose $f:[0, \infty) \rightarrow \mathbf{C}$ is a Lipschitz map, with $f(0)=0$. We define a map $\Omega: \ell_{2} \rightarrow \omega$ by

$$
\Omega_{f}(x)(n)=x(n) f\left(\log \frac{\|x\|_{2}}{|x(n)|}\right)
$$

1991 Mathematics Subject Classification. 46B03.

Supported by NSF grant DMS-9201357 
Here we interpret the right-hand side to be zero if $x(n)=0$. We then define $Z_{2}(f)$ to be the space of pairs $(x, y) \in \ell_{2} \times \omega$ so that

$$
\|(x, y)\|_{f}=\|x\|_{2}+\left\|y-\Omega_{f}(x)\right\|_{2}<\infty .
$$

It then follows that $Z_{2}(f)$ is a Banach space under a norm equivalent to the quasinorm \|\|$_{f}$. Such spaces were first considered in [2], where only the real case was considered; however, the switch to complex scalars, and complex-valued $f$ is routine.

If $s \in \ell_{\infty}$ with $\|s\|_{\infty} \leq 1$ then we have the estimate $\left\|\Omega_{f}(s x)-s \Omega_{f}(x)\right\|_{2} \leq C_{0}\|x\|_{2}$ where $C_{0}$ depends on the Lipschitz constant of $f$ and this leads to fact that there is a constant $C$ such that $\|(s x, s y)\|_{f} \leq C\|(x, y)\|_{f}$. It follows that the spaces $E_{n}$ spanned by $\left(e_{n}, 0\right)$ and $\left(0, e_{n}\right)$ form a UFDD for $Z_{2}(f)$. This UFDD has a certain symmetry, which will be used frequently, for if $\pi: \mathbf{N} \rightarrow \mathbf{N}$ is a permutation and $x_{\pi}(n)=x(\pi(n))$ then $\left\|\left(x_{\pi}, y_{\pi}\right)\right\|_{f}=\|(x, y)\|_{f}$.

We will now specialize to the functions $f_{\alpha}(t)=t^{1+i \alpha}$ for $-\infty<\alpha<\infty$. We write $Z_{2}(\alpha)=Z_{2}\left(f_{\alpha}\right)$ and $\Omega_{\alpha}$ in place of $\Omega_{f_{\alpha}}$.

The following observation is trivial:

Proposition 1. The complex conjugate of $Z_{2}(\alpha)$ is isomorphic to $Z_{2}(-\alpha)$.

Theorem 2. Suppose $Z_{2}(\alpha)$ and $Z_{2}(\beta)$ are isomorphic. Then $\alpha=\beta$.

Proof. We suppose that $\alpha \neq 0$ and that $Z_{2}(\alpha)$ is isomorphic to $Z_{2}(\beta)$. Let $a=1+i \alpha$ and $b=1+i \beta$.

We first observe the following inequalities for $t>s \geq 0$ :

$$
\begin{aligned}
\left|t^{b}-s^{b}\right| & \geq t-s \\
\left|t^{b}-s^{b}\right| & \leq|b|(t-s) \\
\left|t^{b}-s^{b}-b s^{b-1}(t-s)\right| & \leq \frac{1}{2}|b||s|^{-1}(t-s)^{2} .
\end{aligned}
$$

For $w \in \ell_{2}$ define $\Omega_{\beta}^{\prime}(w)(n)=w(n)\left(\log |w(n)|^{-1}\right)^{b}$. Note that

$$
\left\|\Omega_{\beta}(w)-\Omega_{\beta}^{\prime}(w)\right\|_{2} \leq|b|\left|\log \|w\|_{2}\right|\|w\|_{2} .
$$

In particular if $\|w\|_{2} \leq 1$

$$
\left\|\Omega_{\beta}(w)-\Omega_{\beta}^{\prime}(w)\right\|_{2} \leq|b| .
$$

If $A$ is a finite subset of $\mathbf{N}$ we will let $\xi_{A}=\sum_{n \in A} e_{n}$.

We will suppose the existence of an operator $T: Z_{2}(\alpha) \rightarrow Z_{2}(\beta)$ such that $\|T\|<1$ and, $c>0$ so that for every $n,\left\|T\left(e_{n}, 0\right)\right\|_{\beta},\left\|T\left(0, e_{n}\right)\right\|_{\beta}>c$. We will say 
that $T$ is admissible if it satisfies these properties, for some $c>0$. Clearly if there is an admissible operator then, by blocking, we can find an admissible operator $T$ and an increasing sequence of integers $\left(p_{n}\right)_{n=0}^{\infty}$ so that for suitable sequences $u, v, w, y \in \omega$ we have, setting $B_{n}=\left\{p_{n-1}+1, \ldots, p_{n}\right\}, T\left(e_{n}, 0\right)=\left(u \xi_{B_{n}}, v \xi_{B_{n}}\right)$ and $T\left(0, e_{n}\right)=\left(w \xi_{B_{n}}, y \xi_{B_{n}}\right)$. Here $u \xi_{B_{n}}=\sum_{k \in B_{n}} u(k) e_{k}$ etc. Henceforward we consider only operators blocked in this way.

Our first objective is to show that we must have $\lim _{n \rightarrow \infty}\left\|w \xi_{B_{n}}\right\|_{2}=0$. Indeed if this is not the case, then loss of generality we may suppose that $T$ satisfies $\left\|w \xi_{B_{n}}\right\|_{2} \geq \delta>0$ for all $n$. For any integer $N$, let $A=\{1,2 \ldots, N\}$. Then $\left\|T\left(0, N^{-1 / 2} \xi_{A}\right)\right\|_{\beta}<1$ and hence

$$
\left\|N^{-1 / 2} \sum_{k=1}^{N} y \xi_{B_{k}}-\Omega_{\beta}\left(N^{-1 / 2} \sum_{k=1}^{N} w \xi_{B_{k}}\right)\right\|_{2} \leq 1
$$

Since $\left\|y \xi_{B_{k}}-\Omega_{\beta}\left(w \xi_{B_{k}}\right)\right\|_{2} \leq 1$, we have

$$
\left\|\Omega_{\beta}\left(N^{-1 / 2} \sum_{k=1}^{N} w \xi_{B_{k}}\right)-N^{-1 / 2} \sum_{k=1}^{N} \Omega_{\beta}\left(w \xi_{B_{k}}\right)\right\|_{2} \leq 1
$$

From this we get, since $\left\|w \xi_{B_{k}}\right\|_{2} \leq 1$,

$$
\left\|\Omega_{\beta}^{\prime}\left(N^{-1 / 2} \sum_{k=1}^{N} w \xi_{B_{k}}\right)-N^{-1 / 2} \sum_{k=1}^{N} \Omega_{\beta}^{\prime}\left(w \xi_{B_{k}}\right)\right\|_{2} \leq 1+2|b| .
$$

This simplifies to

$$
\frac{1}{N} \sum_{k=1}^{N} \sum_{j \in B_{k}}\left|w_{j}\right|^{2} \mid\left(\log \left(\frac{\sqrt{N}}{|w(j)|}\right)^{b}-\left(\left.\log \left(\frac{1}{|w(j)|}\right)^{b}\right|^{2} \leq(1+2|b|)^{2} .\right.\right.
$$

From this we deduce, using (1), that

$$
\frac{1}{N} \sum_{k=1}^{N} \sum_{j \in B_{k}}\left|w_{j}\right|^{2}(\log \sqrt{N})^{2} \leq(1+2|b|)^{2}
$$

which in turn implies that

$$
\delta^{2}(\log \sqrt{N})^{2} \leq(1+2|b|)^{2}
$$

for all $N$, leading to a contradiction. We may thus suppose that $\lim _{n \rightarrow \infty}\left\|w \xi_{B_{n}}\right\|_{2}=$ 0 . 
It now follows, by passing to a subsequence and rearranging that we can further suppose that $w=0$. We then have $c \leq\left\|y \xi_{B_{n}}\right\|_{2} \leq 1$ for all $n$, and some $c>0$.

We next show again by contradiction that we cannot have inf $\left\|u \xi_{B_{n}}\right\|_{\infty}=0$. Indeed, in the contrary case, we can assume that for any $M$ there exists an admissible $T=T_{M}($ with $w=0)$ so that $\|u\|_{\infty} \leq e^{-M}$.

Under this assumption suppose that $n_{1}<N_{1}$ and $n_{2}<N_{2}$ are pairs of integers and let $\sigma_{r}=\frac{1}{2} \log n_{r}$ and $\tau_{r}=\frac{1}{2} \log N_{r}$ for $r=1,2$. Let $N$ be any integer greater than $\max \left(N_{1}, N_{2}\right)$. Suppose $A$ is a subset of $\{1,2, \ldots, N\}$ with $|A|=n_{1}$. Then $\left\|\left(\xi_{A}, \sigma_{1}^{a} \xi_{A}\right)\right\|_{\alpha}=n_{1}^{1 / 2}$. It follows that

$$
\left\|n_{1}^{-1 / 2} \sigma_{1}^{a} \sum_{k \in A} y \xi_{B_{k}}+n_{1}^{-1 / 2} \sum_{k \in A} v \xi_{B_{k}}-\Omega_{\beta}^{\prime}\left(n_{1}^{-1 / 2}\left(\sum_{k \in A} u \xi_{B_{k}}\right)\right)\right\|_{2} \leq 1+|b| .
$$

For ease of notation let $\rho_{j}=-\log |u(j)|$ when $|u(j)| \neq 0$ and let $\rho_{j}=M$ otherwise; note that $\rho_{j} \geq M$. We can rearrange the preceding equation as

$$
\frac{1}{n_{1}} \sum_{k \in A} \sum_{j \in B_{k}}\left|\sigma_{1}^{a} y(j)+v(j)-\left(\sigma_{1}+\rho_{j}\right)^{b} u(j)\right|^{2} \leq(1+|b|)^{2} .
$$

By averaging we obtain

$$
\frac{1}{N} \sum_{k=1}^{N} \sum_{j \in B_{k}}\left|\sigma_{1}^{a} y(j)+v(j)-\left(\sigma_{1}+\rho_{j}\right)^{b} u(j)\right|^{2} \leq(1+|b|)^{2} .
$$

Similarly we obtain

$$
\frac{1}{N} \sum_{k=1}^{N} \sum_{j \in B_{k}}\left|\tau_{1}^{a} y(j)+v(j)-\left(\tau_{1}+\rho_{j}\right)^{b} u(j)\right|^{2} \leq(1+|b|)^{2}
$$

At this point we use the estimate (3):

$$
\left|\left(s+\rho_{j}\right)^{b}-\rho_{j}^{b}-b s \rho_{j}^{b-1}\right| \leq \frac{|b| s^{2}}{2 M} .
$$

Thus

$$
\left|\left(\tau_{1}+\rho_{j}\right)^{b}-\left(\sigma_{1}+\rho_{j}\right)^{b}-b\left(\tau_{1}-\sigma_{1}\right) \rho_{j}^{b-1}\right| \leq \frac{|b| \tau_{1}^{2}}{M} .
$$

We thus obtain by the triangle law that

$$
\frac{1}{N} \sum_{k=1}^{N} \sum_{j \in B_{k}}\left|\frac{\tau_{1}^{a}-\sigma_{1}^{a}}{\tau_{1}-\sigma_{1}} y(j)-b \rho_{j}^{i \beta} u(j)\right|^{2} \leq \frac{\left(M^{-1}|b| \tau_{1}^{2}+4+4|b|\right)^{2}}{\left(\tau_{1}-\sigma_{1}\right)^{2}}
$$


using the fact that $\left\|u \xi_{B_{k}}\right\|_{2} \leq 1$ for all $k$.

Using a similar calculation for $\sigma_{2}, \tau_{2}$ and using the fact that $\frac{1}{N} \sum_{k=1}^{N} \sum_{j \in B_{k}}|y(j)|^{2} \geq$ $c^{2}$ we obtain that

$$
\left|\frac{\tau_{1}^{a}-\sigma_{1}^{a}}{\tau_{1}-\sigma_{1}}-\frac{\tau_{2}^{a}-\sigma_{2}^{a}}{\tau_{2}-\sigma_{2}}\right| \leq K_{1}+K_{2}
$$

where

$$
K_{r}=\frac{1}{c}\left(\frac{M^{-1}|b| \tau_{r}^{2}+4+4|b|}{\tau_{r}-\sigma_{r}}\right) .
$$

Now $M$ is arbitrary, and $\tau_{r}, \sigma_{r}$ are restricted only to be of the form $\frac{1}{2} \log m$ where $m \in \mathbf{N}$. It thus follows that for any $\kappa>1$ such that $\kappa^{a} \neq 1$ we must have that

$$
\lim _{\sigma \rightarrow \infty} \frac{\kappa^{a} \sigma^{a}-\sigma^{a}}{\kappa \sigma-\sigma}=\lim _{\sigma \rightarrow \infty} \sigma^{i \alpha} \frac{\kappa^{a}-1}{\kappa-1}
$$

exists, which contradicts the fact that $\alpha=\Re a \neq 0$.

Our conclusion is then there exists an admissible $T$ so that inf $\left\|u \xi_{B_{n}}\right\|_{\infty}>0$. Under these circumstances we can apply the diagonalization procedure of Proposition 1.c.8 of [3] and a subsequence argument to produce an operator $S: Z_{2}(\alpha) \rightarrow Z_{2}(\beta)$ with $\|S\|<1$ and so that $S\left(e_{n}, 0\right)=\left(\lambda e_{n}, \mu e_{n}\right)$ and $S\left(0, e_{n}\right)=\left(0, \nu e_{n}\right)$ with $\lambda \neq 0$. Let $A=\{1,2 \ldots, N\}$. Then $\left\|S\left(N^{-1 / 2} \xi_{A}, \sigma^{a} N^{-1 / 2} \xi_{A}\right)\right\|_{2}<1$ where $\sigma=\frac{1}{2} \log N$. Hence,

$$
\left|\nu \sigma^{a}-\lambda(\sigma+\log |\lambda|)^{b}+\mu\right| \leq 1
$$

As this holds for all $N$ we must have $a=b$ or $\alpha=\beta$, as required.

Corollary 3. The space $Z_{2}(\alpha)$ is not isomorphic to its complex conjugate when $\alpha \neq 0$.

\section{REFERENCES}

1. J. Bourgain, Real isomorphic complex Banach spaces need not be complex isomorphic, Proc. Amer. Math. Soc. 96 (1986), 221-226.

2. N.J. Kalton and N.T. Peck, Twisted sums of sequence spaces and the three space problem, Trans. Amer. Math. Soc. 255 (1979), 1-30.

3. J. Lindenstrauss and L. Tzafriri, Classical Banach spaces I, Sequence spaces, Springer, Berlin, 1977.

4. P. Mankiewicz, A superreflexive Banach space $X$ with $\mathcal{L}(X)$ admitting a homorphism into the Banach algebra $C(\beta \mathbf{N})$, Israel J. Math. 65 (1989), 1-16.

5. S.J. Szarek, On the existence and uniqueness of complex structure and spaces with "few" operators, Trans. Amer. Math. Soc. 293 (1986), 339-353.

Department of Mathematics, University of Missouri Columbia, MO 65211, U.S.A. E-mail address: mathnjk@mizzou1.bitnet 